\title{
Biogeochemical behaviour of anionic radionuclides in soil: Evidence for biotic interactions
}

\author{
L. Février and A. Martin-Garin \\ Laboratory of Radioecology and Ecotoxicology, Institute for Radioprotection and Nuclear \\ Safety, Cadarache, BP. 3, 13115 Saint Paul-lez-Durance Cedex, France
}

\begin{abstract}
Tc and ${ }^{79}$ Se, two long-lived radionuclides, are supposed to be highly mobile in soils, because of their anionic forms. Their behaviours in the soil are often considered only from a physico-chemical point of view, although the microorganisms can affect either directly or indirectly their speciation. This study demonstrates the role of the microbial compartment in the retention of Se and Tc in soil by comparing experiments with soils constrained to different microbiological status (sterile / raw / amended). Kd coefficients for Se and Tc were determined in batch experiments, whereas transport of Se and Tc was investigated through column leaching experiments. Kd for Se was enhanced for the raw soil without amendment compared to the value obtained for the sterilised soil. The retention of Se was higher again in the amended soil. Besides, a biofilm, which can directly retain Se, was obtained at the entrance of the amended soil column. This effect was less obvious for Tc in batch experiments, but was revealed by leaching experiments where a high quantity of Tc was retained in the amended soil columns. These results give strong evidence that microorganisms are responsible for a greater retention of Se and Tc in soil.
\end{abstract}

\section{INTRODUCTION}

The behaviour of radionuclides in soil is controlled by various physical, chemical, and biological processes that may induce their immobilisation in the media or their migration out of the media (including volatilization, transport, or root-transfer). The physico-chemical processes involve both mechanisms in solution (as a function of $\mathrm{pH}$, redox potential, etc.) and at the liquid-solid interface (adsorption, precipitation, etc.). They depend mostly on the intrinsic properties of the soil. On the other hand, the biotic processes are often neglected. Yet, in surface horizons, the microbiological processes induced by the plant roots and the microorganisms themselves cannot be ignored.

Selenium and technetium are two of a number of redox-sensitive radionuclides of importance (others include uranium, and neptunium) in assessments of the performance of high-level radioactive waste repositories [1]. ${ }^{79}$ Se and ${ }^{99} \mathrm{Tc}$, which half-lives are respectively $1.13 \times 10^{6}$ and $2.13 \times 10^{5}$ years [2], are fission products. They both exist in many oxidation states and in a variety of inorganic and organic species. In moderately oxidizing conditions like surface soils, selenite (Se(IV)) and pertechnetate (Tc(VII)) are the most environmentally relevant forms of these elements. They are mainly present in their anionic $\left(\mathrm{HSeO}_{3}{ }^{-}\right.$and $\mathrm{SeO}_{3}{ }^{2-}, \mathrm{TcO}_{4}{ }^{-}$) or neutral forms (e.g. $\mathrm{CaSeO}_{3}{ }^{0}$ in calcareous soils). In these forms, Se(IV) is assumed to be mobile at the near-neutral to alkaline $\mathrm{pH}$ values typical of a large number of soils although immobilisation may be caused by different mechanisms among which adsorption, precipitation, co-precipitation or absorption by microorganisms are usually reported. Tc(VII) is known to show poor ligand-complexing capabilities and to be weakly sorbed under most geochemical conditions. Therefore Tc(VII) is supposed to be highly mobile and to migrate rapidly through the vadose zone and in the groundwater. 
The redox chemistry of Se and Tc is crucial in governing their mobility in soil. Several studies have shown that reduction of Se(IV) and Tc(VII) to insoluble forms, as Se(0) or Tc(IV), are efficient in their immobilisation. These reduction reactions can be achieved abiotically by heterogeneous reactions with FeII-containing minerals for example [3,4]. But they have also been proved to be microbially mediated, resulting either from direct interaction between Se / Tc and some microorganisms [5, 6, 7, 8] or from physico-chemical changes due to the microbial activity [4, 9].

The study presented here is part of a wider research program which aims (1) at defining the limitation (or not) of the use of the concept of Kd to describe the mobility of Se(IV) and Tc(VII) in agricultural soils and (2) at assessing the importance of microbiological interactions in the retention processes. Laboratory experiments were performed to evaluate the importance of microbiological interactions in the retention of $\mathrm{Se}(\mathrm{IV})$ and $\mathrm{Tc}(\mathrm{VII})$ in two agricultural soils. The role of the microbiological component in the retention of Se and Tc was investigated by performing experiments with soils constrained to contrasted microbial states (raw, sterile, amended or anaerobic-conditioned soils). The experiments were carried out either in batch (static investigations) or in column devices (dynamic investigations).

\section{MATERIAL AND METHODS}

\subsection{Soil, radionuclides and amendments}

Samples from the upper layer of two natural soils with contrasting physico-chemical properties (pH, carbonate and clay contents) were employed. Soil 1 was a silty-loam soil, with a high organic matter content (3.2 \%) and an acidic $\mathrm{pH}$ (6.5). Soil 2 was a silty-clay soil, with a lower organic matter content (2.5\%) and a basic $\mathrm{pH}(8.2)$ due to a high amount of carbonate (39.2\%). Both soils were sieved at $2 \mathrm{~mm}$ and re-wetted up to $20 \%(\mathrm{~m} / \mathrm{m})$ before experiments to ensure an homogeneous microbial activity.

Selenium and Technetium were used in solutions, respectively as selenite -Se(IV)- and pertechnetate -Tc(VII). ${ }^{75} \mathrm{Se}(\mathrm{IV})$ was added to stable Se(IV) carrier, yielding to a total concentration of $100 \mu \mathrm{g} . \mathrm{L}^{-1}$ for all experiments. Tc(VII) solution consisted of ${ }^{99} \mathrm{Tc}$ or ${ }^{95 \mathrm{~m}} \mathrm{Tc}$ at $500 \mu \mathrm{g} . \mathrm{L}^{-1}$.

Glucose and nitrate were used as soluble amendments to stimulate the microbial activity of both soils. They were used at equi-molar concentration of $10^{-3} \mathrm{~mol} \mathrm{~L}^{-1}$ when added to soil 1 and of $5.6 \times 10^{-3}$ mol. $\mathrm{L}^{-1}$ when added to soil 2 . Besides the amendment of the solutions, some of them were degassed in order to obtain anaerobic condition.

For experiments with no microbial activity, sterilisation was achieved by gamma irradiation for both soils and by filtration at $0.22 \mu \mathrm{m}$ for all solutions.

\subsection{Bio-physico-chemical analyses}

The monitoring of the soil microbiological state was carried out by respiration monitoring during 48 hrs with Oxitop ${ }^{\circledR}$ device and by bacterial counts on agar plate.

${ }^{75}$ Se and Tc concentrations were measured by liquid scintillation (Wallas Quantulus), in the bulk solution and in the solution-fraction under $0.22 \mu \mathrm{m}$ (size cut allowing the microorganisms to be separated from the bulk solution).

Chemical composition of all solutions was checked for major anions and cations, as well as for glucose and nitrate.

\subsection{Batch experiments - Kd determination}

Batch experiments were carried out in polycarbonate centrifuge tubes, by mixing the dry soil and the radioactive solutions containing or not the amendments $(\mathrm{m} / \mathrm{V}=1 / 5)$. A partition coefficient $(\mathrm{Kd})$ for Se and Tc sorption was calculated according to the following equation: 


$$
K d=\frac{\left(A_{i}-A_{f}\right) \cdot V}{A_{f} \cdot m}
$$

with $A_{i}$ and $A_{f}$ the initial and the final radionuclide activities in the batch supernatants (Bq. $\left.L^{-1}\right)$, $m$ and $\mathrm{V}$ the dry soil $(\mathrm{kg})$ and the solution $(\mathrm{L})$ content respectively.

Contact time for batch experiments varied from $1 \mathrm{hr}$ to 1 month according to the conditions tested.

\subsection{Column experiments}

Experiments were carried out by percolating pulses of Se and Tc in non-contaminated saturated soil columns amended or not with glucose and nitrate. All experiments were realised at constant flow velocity and steady state. Hydrodynamic properties of the soil columns were characterised by the use of two flow tracers: tritiated water $\left({ }^{3} \mathrm{H}_{2} \mathrm{O}\right)$ and chloride $\left({ }^{36} \mathrm{Cl}^{-}\right)$as an anionic flow tracer. Retardation of Se and Tc were investigated by comparing their breakthrough curves (BTC) at the outlet of the column to the inert tracers BTC (Fig. 1). At the end of the experiments, retention profiles in the soil columns were determined for each radionuclide to appreciate the extent of their migration.

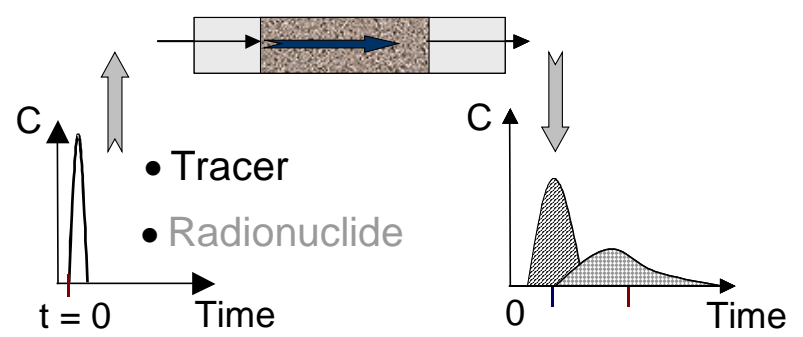

Figure 1. Schematic diagram showing the column experiments.

\section{RESULTS AND DISCUSSIONS}

\subsection{Microflora stimulation}

Stimulation of the soil microflora was satisfactorily achieved by the use of glucose and nitrate. Adding glucose to soil 2 always led to an enhancement of its respiratory activity (Fig. 2), but only the addition of glucose and nitrate together yield to a significant increase of the number of cultivable bacteria (Fig. 3).

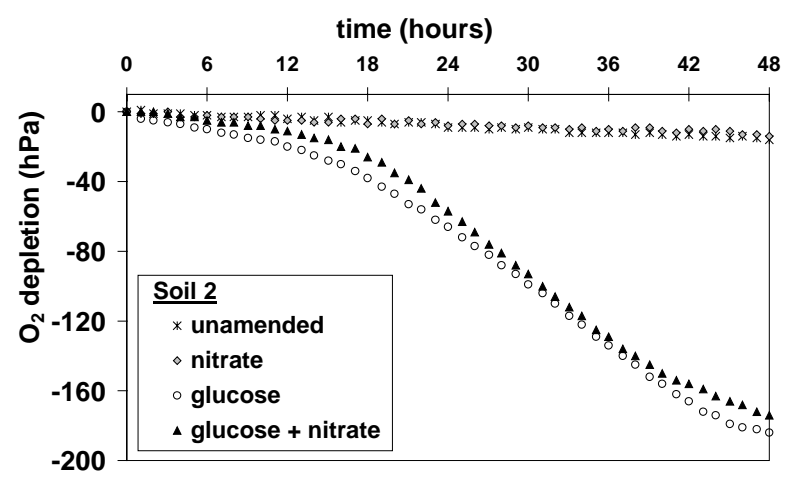

Figure 2. Respiratory activity $\left(\mathrm{O}_{2}\right.$ consumption) of soil 2 in Oxitop ${ }^{\circledR}$. 


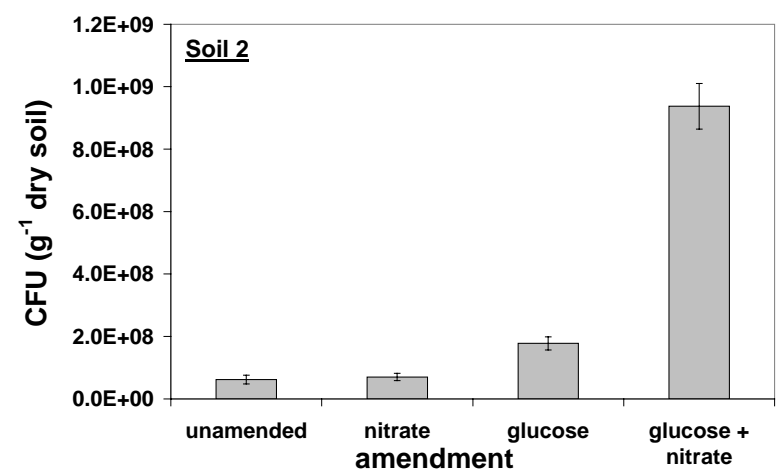

Figure 3. Bacterial counts in soil 2 after 48 hrs in Oxitop ${ }^{\circledR}$.

\subsection{Batch experiments}

As shown by the batch experiments, Se and Tc retention were kinetically limited according to the soil's microbiological state. Results with sterile soil were taken as a reference for the contribution of pure abiotic processes in Se and Tc sorption. According to the Kd coefficients at 48 hrs (Fig. 4 and 6), Se and Tc showed a contrasted behaviour towards abiotic retention. While Se was moderately retained

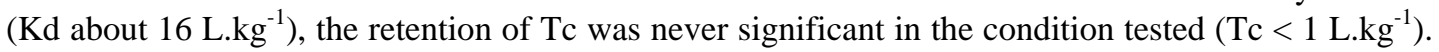
These findings were in good agreement with previous studies on these elements [10, 11]. The role of the microorganisms in the retention of Se and Tc occurred in different conditions depending on the type of amendments used and on the duration of the experiment.

After 48 hrs, Kd coefficient of Se was multiplied by a factor of 6 in the microbially active soil stimulated by glucose and nitrate (Fig. 4) both in microaerobic (non-degassed) and in anaerobic (degassed) conditions. Moreover, in these cases, $40 \%$ of the Se in the batch supernatants were recovered in the fraction $>0.2 \mu \mathrm{m}$, suggesting a direct link between Se and microorganisms.

The effect of the microbial activity of the raw soil on Se retention compared to the sterile soil was more significant for longer contact-time. Kd coefficient with the raw soil increased from $22 \mathrm{~L}^{\mathrm{kg}} \mathrm{kg}^{-1}$ at 48 hrs to $50{\mathrm{~L} . \mathrm{kg}^{-1}}^{-1} 28$ days; whereas no such kinetic effect was seen with the sterile soil (Fig. 5). As it was hypothesized that long-term batch experiment with a microbially active soil could have led to physico-chemical changes in the batch (reducing / anaerobic condition), it was impossible to correlate the increase of the Kd value to direct interactions between Se and microorganisms.

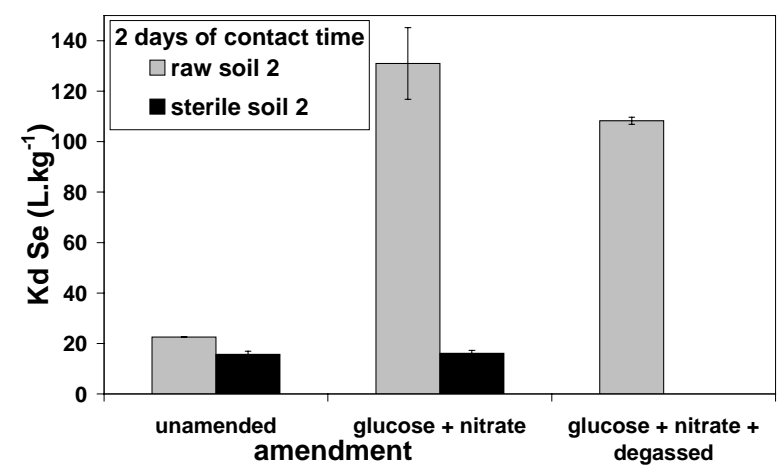

Figure 4. Se Kd coefficient for soil 2 with various amendment after 2 days of contact time. 


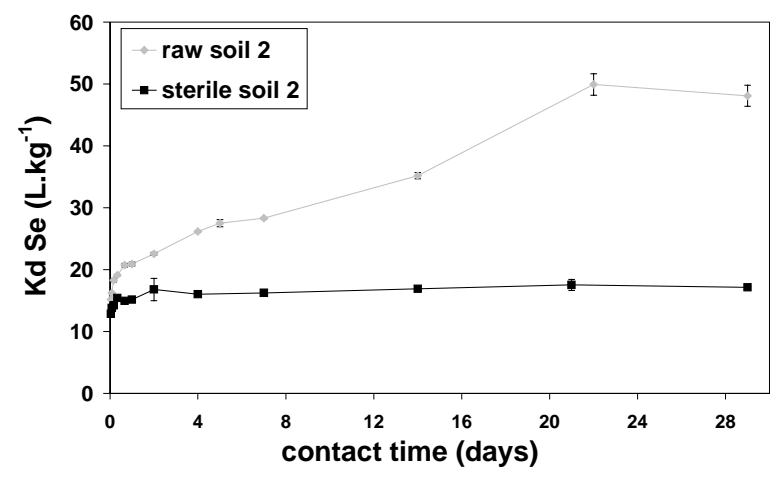

Figure 5. Development in time of Se Kd coefficient for soil 2.

Unlike Se, a large increase of Kd value for Tc $\left(130 \mathrm{~L} . \mathrm{kg}^{-1}\right)$ was achieved with the soil stimulated by glucose and nitrate, only after 7 days of contact-time in anaerobic condition (Fig. 7). These results emphasized the need of reducing environment for Tc to be retained, suggesting a possible reduction of the pertechnetate ion. Yet to correlate this result to a direct or an indirect effect of the microbial activity of the soil was difficult. Contrary to Se, microbially-mediated reduction of Tc(VII) was proved only in anaerobic condition [7,8]. But abiotic reduction of Tc(VII) should not be excluded [4].

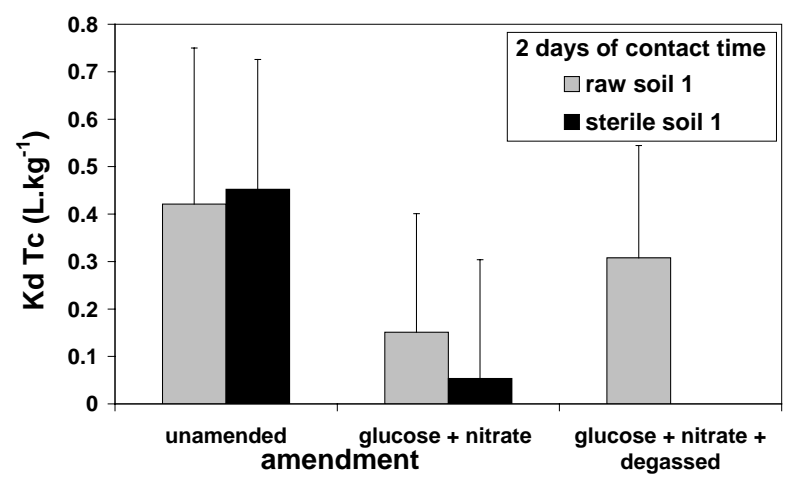

Figure 6. Tc Kd coefficient for soil 1 with various amendments after 2 days of contact time.

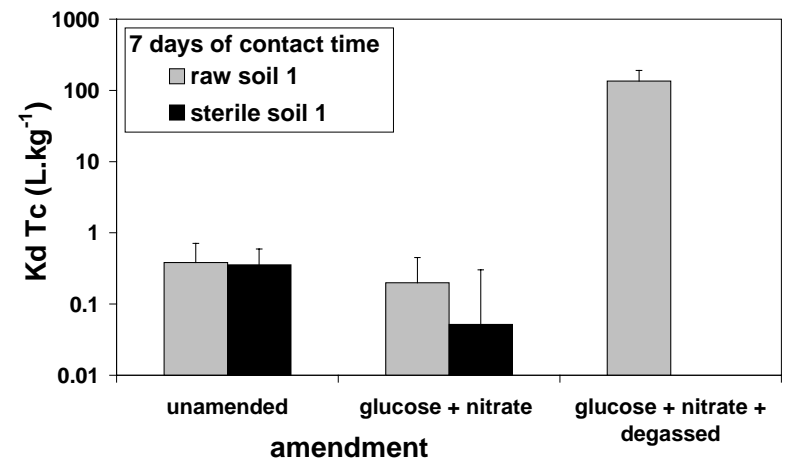

Figure 7. Tc Kd coefficient for soil 1 with various amendments after 7 days of contact time. 


\subsection{Column experiments}

In most of the experiments, Se was never eluted at the outlet of the soil column. Sometimes a small part of Se was eluted at the beginning of the experiment, with the same velocity as water tracers. This result was attributed to colloidal transport of Se (Fig. 8). Se retention profile in the raw soil showed an accumulation of Se in the first $5 \mathrm{~cm}$ of the column and few migration in the deeper horizons (data no shown). This low migration, which may have resulted from the kinetic interactions involved in a microbially active raw soil, showed the inadequacy of a simple Kd transport model to predict Se migration in soil horizons. Evidences of direct interactions between Se and soil microorganisms were obtained with the column amended with glucose and nitrate. These nutrients rapidly led to the development of a biofilm at the entrance of the column, which had retained most of the Se injected (Fig. 9).

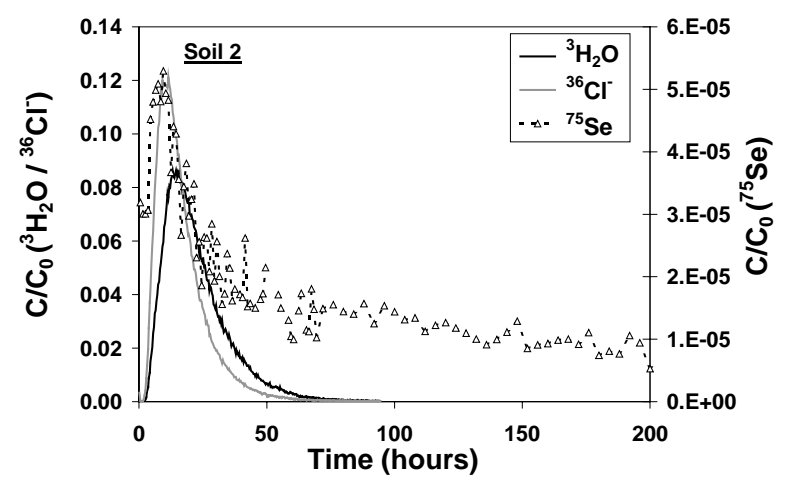

Figure 8. Breakthrough curves at the outlet of the raw soil column.

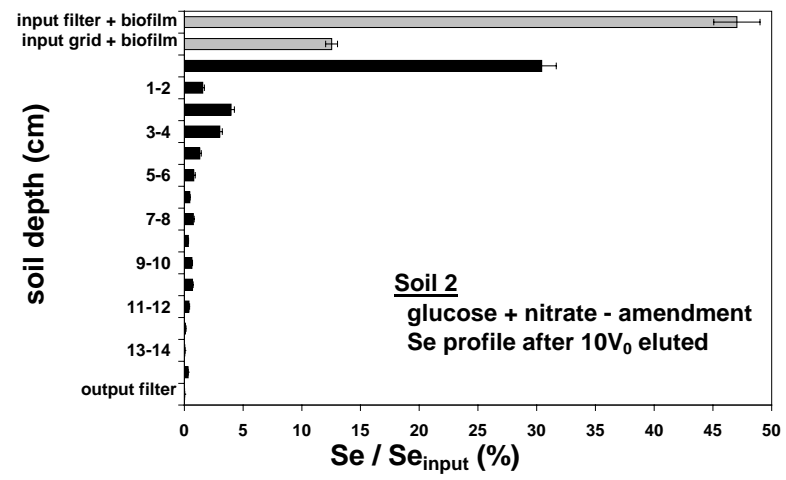

Figure 9. Se retention profile in the soil column amended with glucose and nitrate.

Tc migration in the raw soil columns was in good agreement with the small Kd value obtained for this condition. Almost all the Tc injected (between 90 and $100 \%$ ) was eluted with no retardation compared to tritium (Fig. 10). The slight difference in the Tc mass balance was correlated to the flow velocity inside the column, i.e. the residence time of Tc in the porous medium, and may have been caused by kinetically limited reactions.

As for batch experiments, addition of glucose and nitrate in anaerobic conditions entailed a greater retention of Tc in the soil column. Surprisingly, this retention led to a decrease in the Tc mass balance but no retardation for the fraction of Tc eluted. A possible explanation for this phenomena lies 
in a partial reduction of Tc(VII) into an insoluble form and the elution of the remaining Tc(VII). This result, which had been occulted in batch experiments, suggests that reduction of Tc(VII) is a preferred mechanism towards adsorption for Tc(VII) retention in soils.

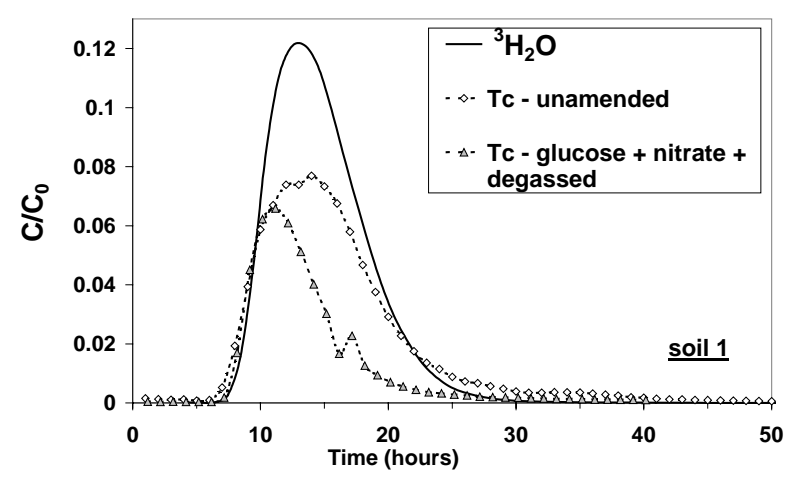

Figure 10. Breakthrough curves at the outlet of the non amended and amended soil column.

Performing only simple batch measurements could have led to a misrepresentation of Se and Tc fates in the soil by underestimating Se and Tc global removal from the solution and totally ignored facilitated transport processes. Biotic's reaction kinetics, which definitely depended on the microbial biomass and activity of the soil are considered to be the key factors responsible of the discrepancies between batch and columns results for Se and Tc. In our experimental set-ups, the soil microbial status were strongly constrained by the organization of the solid substrate that differed significantly from homogeneous soil/solution suspensions to well structured porous media. These two types of organization may have a great influence on the microbial development strategy leading to various microbial, solid and liquid interfaces [12]. Thus, combining both static and dynamic experiments allowed to check the accuracy of biotic and abiotic sorption parameters to characterize radionuclide mobility.

\section{CONCLUSION}

These experiments showed that Se and Tc behaviours in soils were dependant on the physico-chemical environment but also on the microbial activity of the soil. Our results gave evidences of direct interaction between Se(IV) and specific microbial communities both in aerobic and in anaerobic conditions, leading to a large increase of the Kd coefficient. Compared to the retention capacity of a sterile soil, soil's microbial activity on a long-time scale resulted obviously in an increase of Se retention. The mechanisms responsible for this retention were not identified and further research is needed. Experiments in soil columns showed very few migration of Se(IV) in all conditions. Thus reduction of Se(IV) in the soil, mediated by the microorganisms or not, can be an explanation to its strong immobilisation.

Retention of Tc in the soil was rather small in aerobic environment. However in anaerobic condition, Tc can be greatly retained either due to direct or indirect microbial activity. In this condition, column experiments gave evidence for the non reduced Tc(VII) forms to migrate rapidly in the soil horizon.

For such elements, which mobility is governed by the soil oxydo-reduction status, transport is rather complex as it is controlled by chemical and microbiological kinetic interactions. Reversibility of the retention mechanisms, undetectable in our experiments even for long time of solid/solution interactions, has still to be assessed in addition to the key factors controlling Se and Tc reduction, precipitation and or sorption processes. Yet a simple Kd transport model seems not capable to predict Se(IV) and Tc(VII) transport as its validity domain appears largely constrained. 


\section{Acknowledgments}

This reserach program was partly funded by Andra (Nuclear Waste Management French Agency) and by the European Community ( $5^{\text {th }}$ Framework Program - BORIS / FIGE-CT-2000-00071).

\section{References}

[1] Koch-Steindl H. and Pröhl G., Radiat. Environ. Biophys. 40 (2001) 93-104.

[2] Songsheng J., Jingru G., Chunsheng L., Anzhi C., Ming H., Shaoyong W. and Shilin L., Nucl. Instr. and Meth. in Phys. Res. B 123 (1997) 405-409.

[3] Myneni S.C.B, Tokunuga T.K. and Brown Jr. G.E., Science 278 (1997) 1106-1109.

[4] Fredrickson J.K., Zachara J.M., Kennedy R.K., McKinley J.P., Heald S.M., Liu G. and Plymale A.E., Geochim. Cosmochim. Acta 68 (2004) 3171-1187.

[5] Garbisu C., Ishii T., Leighton T. and Buchanan B.B., Chem. Geol. 132 (1996) 199-204.

[6] Oremland R.S., Herbel M.J., Switzer Blum J., Langley S., Beveridge T.J., Ajayan P.M., Sutto T., Ellis A.V. and Curran S. Appl. Environm. Microbiol. 70 (2004) 52-60.

[7] LLoyd J.R., Cole J.A. and Macaskie L.E., J. Bacteriol. 179 (1997) 2014-2021.

[8] Henrot J., Health Physics 57 (1989) 239-245.

[9] LLoyd J.R., Sole V.A., Van Praagh C.V.G. and Lovley D.R., Appl. Environm. Microbiol. 66 (2000) 3743-3749.

[10] Dhillon K.S. and Dhillon S.K. Geoderma 93 (1999) 19-31.

[11] Sheppard S.C., Sheppard M.I. and Evenden W.G., J. Environ. Radioactivity 11 (1990) 215-233.

[12] Pallud C., Deschesne A., Gaudet J.-P., Debouzie D. and Grundmann G.L., Appl. Environm. Microbiol. 70 (2004) 2709-2716. 